We will then re-audit the data before and after feedback cycles to monitor for any reduction in prescribing errors.

\section{G150(P) FORWARD THINKING: SEEKING ALTERNATIVES TO BLEEPS}

${ }^{1} \mathrm{D}$ Roberts, ${ }^{2} \mathrm{~S}$ Webb, ${ }^{3} \mathrm{AL}$ Newnham. ' General Paediatrics, Leeds Children's Hospital, Leeds, UKi ${ }^{2}$ Paediatric Surgery, Leeds Children's Hospital, Leeds, UKi ${ }^{3}$ Paediatric Nephrology, Leeds Children's Hospital, Leeds, UK

\subsection{6/archdischild-2020-rcpch. 121}

Introduction 'Bleep' systems are a central communication tool between medical and nursing teams in hospital. The majority of bleeps are for non-urgent tasks, this inefficient use of the bleep system leads to routine tasks take longer to complete and disruption of patient care. The Health and Social Care Secretary ordered pagers for non-emergency communications be removed from the NHS by 2021 .

Objective Reduction in the number of bleeps for non-urgent tasks in a tertiary paediatric hospital.

Methods A multi-professional working group was established and developed a standard operating procedure (SOP) for bleeping including a 'traffic light' system with non-urgent tasks (completion within 1-4hrs) categorized as green. A job book with designated time slots for medical visits to each ward was introduced. A number of PDSAs (Plan, Do, Study, Act) cycles were completed using the job books but were ultimately unsuccessful. Therefore, a new direction using an electronic solution was explored. The FORWARD app (a secure messaging platform) was chosen to be piloted on two wards. Baton phones pre-installed with FORWARD were given to the medical and nursing teams alongside training. Communication via the platform was limited to non-urgent tasks and urgent tasks remained via the bleep system.

Results Telecommunications records from two weeks either side of implementation of FORWARD app demonstrated a significant reduction in the number of bleeps, the median decreasing from 21 to 12 (run chart available). The feedback was positive, and the nurses reported doctors responded more quickly on FORWARD. The main challenge was inconsistent wireless internet connection, affecting acknowledgment of tasks.

Conclusion This project has shown that a novel, secure messaging platform can effectively reduce the number of nonurgent bleeps. This requires an appropriate induction to the platform, a SOP on the use of the platform/bleeps and adequate IT infrastructure to ensure reliability and safety. This trial has also highlighted the importance of careful consideration of the governance implications when using patient identifiable data which is a clinical governance requirement for patient safety. Collaboration with the trust digital informatics and information governance team has led to the development of acceptable user policies to guide this.

\section{G151(P) IMPROVING NUTRITIONAL INTAKE IN EXTREMELY PRETERM INFANTS IN WEEK ONE}

${ }^{1} \mathrm{HM}$ Cobb, ${ }^{1} \mathrm{C}$ Turner, ${ }^{2} \mathrm{~S}$ Bates, ${ }^{3} \mathrm{TA}$ Warlow, ${ }^{1} \mathrm{PA}$ Mannix. ${ }^{1}$ Southmead Neonatal Intensive Care Unit, North Bristol NHS Trust, Bristol, UK; ${ }^{2}$ Women's and Children's, Great Western Hospitals NHS FT, Swindon, UK; ${ }^{3}$ All Wales Paediatric Palliative Care Network, Ty Hafan Children's Hospice, Cardiff, UK
Aim Optimised early nutrition in extremely preterm infants is associated with improved developmental outcome, but the delivery of parenteral nutrition (PN) must often be balanced against the need for multiple drug infusions.

A 2012 audit demonstrated that our unit was not reaching targets for energy and protein intake in the first week of life in infants $<28$ weeks gestation. We aimed to improve in line with national guidelines by making changes to PN.

Methods New PN was formulated by a multi-disciplinary team including specialist dieticians and pharmacists. This was concentrated to run at maximum $100 \mathrm{ml} / \mathrm{kg} / \mathrm{d}$, with lipid at 20 $\mathrm{ml} / \mathrm{kg} / \mathrm{d}$, allowing drug infusions to take a larger proportion of the fluid requirement without titrating from PN volume. Additionally, a higher threshold for stopping lipids was agreed (serum triglyceride levels $\geq 4 \mathrm{mmol} / \mathrm{L}$ ).

25 inborn babies born at $<28$ weeks from May 2018 to May 2019 were included (6 were excluded due to death or incomplete notes). Notes were reviewed retrospectively, and delivery of $\mathrm{PN}$, lipid, feeds and dextrose infusions were recorded for the first 7 days. Total calorie and protein intake were then calculated.

Results Energy intake increased compared to 2012, with a mean additional $10.2 \mathrm{kcal} / \mathrm{kg} / \mathrm{d}$ on day 1 , and $23.36 \mathrm{kcal} / \mathrm{kg} / \mathrm{d}$ on day 7 , which is closer to recommendations (table 1 ).

Abstract G151(P) Table 1 Calorie intake (kcal/kg/d)

\begin{tabular}{llllllll}
\hline Day & $\mathbf{1}$ & $\mathbf{2}$ & $\mathbf{3}$ & $\mathbf{4}$ & $\mathbf{5}$ & $\mathbf{6}$ & $\mathbf{7}$ \\
\hline 2019 & 46.73 & 64.09 & 77.32 & 86.06 & 90.68 & 93 & 97.48 \\
2012 & 36.51 & 52 & 61.79 & 66.08 & 70.72 & 74.48 & 74.12 \\
Recommended & $60-80$ & $80-$ & $80-$ & $100-$ & $100-$ & $100-$ & $100-$ \\
(BAPM 2016) & & 100 & 100 & 120 & 120 & 120 & 120 \\
\hline
\end{tabular}

Protein intake was also higher, and was within the recommended range from day 3 (table 2).

Abstract G151(P) Table 2 Protein intake $(\mathrm{g} / \mathrm{kg} / \mathrm{d})$

\begin{tabular}{llllllll}
\hline Day & $\mathbf{1}$ & $\mathbf{2}$ & $\mathbf{3}$ & $\mathbf{4}$ & $\mathbf{5}$ & $\mathbf{6}$ & $\mathbf{7}$ \\
\hline 2019 & 1.81 & 2.48 & 2.9 & 3.25 & 3.43 & 3.46 & 3.5 \\
2012 & 1.48 & 2.03 & 2.42 & 2.56 & 2.84 & 2.92 & 2.84 \\
Recommended & $2-2.5$ & $2.7-$ & $2.7-$ & $2.7-$ & $2.7-$ & $2.7-$ & $2.7-$ \\
(BAPM 2016) & & 3.5 & 3.5 & 4.0 & 4.0 & 4.0 & 4.0 \\
\hline
\end{tabular}

Conclusion Use of a more concentrated PN solution alongside higher thresholds for stopping lipid infusions has resulted in greater early energy and protein intakes in infants $<28$ weeks in our unit.

\section{G152(P) A QUALITY IMPROVEMENT PROJECT FOR MANAGEMENT OF WELL BABIES WITH JAUNDICE IN A DISTRICT GENERAL HOSPITAL}

1J Coburn, ${ }^{2} \mathrm{~J}$ Martin, ${ }^{2} \mathrm{~A}$ Chavan, ${ }^{1} \mathrm{~S}$ English, ${ }^{2} \mathrm{~A}$ Hickey, ${ }^{2} \mathrm{R}$ Gandhi. ${ }^{1}$ Midwifery Department, Princess Royal University Hospital, King's College Hospital NHS FT, London, UK; ${ }^{2}$ Neonatal Unit, Princess Royal University Hospital, King's College Hospital NHS FT, London, UK 
Background An audit performed on the management pathway of well babies with jaundice referred from community demonstrated long waiting times in A\&E and on the local SCBU, with pathways of care not clarified. This posed an increased risk for babies being exposed to infection in $A \& E$ and increased pressure on nursing and medical staff. In view of this, a new midwifery led pathway was implemented in May 2019. This has clear guidelines and SOP for referrals and exclusions by the community team and escalation pathways to medical staff. Well babies are assessed in a walk in clinic by midwives using transcutaneous bilirubinometers (TCB) in the maternity assessment unit (MAU). Further referral to paediatric staff are made if they fulfil specific high risk criteria or bilirubinometer reading is $>250 \mathrm{umol} / \mathrm{l}$. Unwell babies are referred directly to $\mathrm{A} \& \mathrm{E}$ for further assessment and management.

Objective

- To determine the safety and effectiveness of midwifery led pathway

- To determine reduction in the waiting times

- To determine cost implications of this service

Method Prospective data was collected for all babies being referred and assessed following the service change. Waiting times and cost effectiveness were analysed and compared to previous pathway.

Results The number of babies per month being assessed by the midwifery team has remained same as that seen previously (Average 72 babies/month). Average age at referral, gestational age and birth weight were similar in both pathways: 3.9 days, 39 weeks GA and 3377 gm respectively. Waiting times reduced significantly from average of 120 minutes to 31 minutes with the new pathway. Only $15 \%$ of the babies assessed in MAU were referred for further assessment to medical team. The one-time cost of purchasing of TCB was $£ 3400$ as compared to cost of blood tests performed in one month was $£ 1499.94$.

Conclusion Midwifery led pathway significantly improved waiting times for babies to be assessed, mitigating the risks of them being exposed to serious infections in A\&E. It is a costeffective and efficient pathway for assessment of well babies. The pathway provides an adequate safety net for unwell babies as well as babies with risk factors being referred appropriately to the medical team.

\section{G153(P) 'IMPROVING EFFICACY OF HYDROXYCARBAMIDE PRESCRIPTIONS IN PAEDIATRIC SICKLE CELL DISEASE'}

${ }^{1}$ VMY Wong-Spracklen, ${ }^{1} \mathrm{~F}$ Kagalwala, ${ }^{2} \mathrm{~A}$ Senosiain-Martinez. ${ }^{1}$ Department of Paediatrics, The Lister Hospital, East and North Hertfordshire Trust, Stevenage, UK; ${ }^{2}$ Pharmacy Department, The Lister Hospital, East and North Hertfordshire NHS Trust, Stevenage, UK

\subsection{6/archdischild-2020-rcpch.124}

Aims Hydroxycarbamide use in children with sickle cell anaemia is on the increase. Given its narrow therapeutic index, close monitoring and careful dose-titration are required. Our project aimed to introduce a reliable, sustainable method of monitoring blood results to facilitate decision-making by the paediatric consultant (PC) with haematology expertise with regards to hydroxycarbamide dose adjustments.

Methods This retrospective cohort study included all paediatric sickle-cell patients from 1 January 2018 until 1 March 2019. In the first quality-improvement (QI) cycle of plan-do-study- act, the PC was responsible for prescribing hydroxycarbamide, checking the patient's blood test results and assessing trends. The clinician had to repeatedly access electronic records to check on results or retrospectively determine at which time point, decision to adjust the dose of Hydroxycarbamide was made. With frequent outpatient blood tests and hospital admissions in the cohort, this format of dose-titration, retrospective referencing and documentation, proved timeconsuming.

In the second QI cycle, a new chart was designed and introduced. On the front of the chart, spaces were allocated for 8 consecutive prescriptions. Spaces for blood results documentation were allocated at the back of the chart. PC and pharmacist were able to refer to the formal charting of blood results within the patient's hydroxycarbamide prescription chart.

Results 15 patients were on hydroxycarbamide (median age 10.9 years, $53.3 \%$ male, $46.7 \%$ female). Since introducing the new prescription chart, frequency of blood testing improved by $75 \%$, from 4 to 7 times annually. Hydroxycarbamide dose changes increased by $300 \%$ from 0.33 times per year to once annually. $67 \%$ of hydroxycarbamide patients showed an increase in $\mathrm{HbF} \%$ to $>25 \%$ within 1 year of using the new prescription charts, indicating maximal haematological response, reducing pain scores and hospital attendances significantly. Mean dose of hydroxycarbamide after 1 year of new chart use was $21 \mathrm{mg} / \mathrm{kg} / \mathrm{d}$ (median $17 \mathrm{mg} / \mathrm{kg} / \mathrm{d}$ ). Due to increased confidence in patient-monitoring, the total patients on hydroxycarbamide increased by $27 \%$, from 15 to 19 , reflecting improved quality of care for patients with sickle cell for the region.

Conclusion By introducing a new hydroxycarbamide chart design, efficiency of dose-optimisation, compliance-checking and communication between sickle cell patients and haematology-care paediatricians were improved.

\section{G154(P) USER SERVICE EVALUATION OF THE REGIONAL PAEDIATRIC BRONCHOSCOPY SERVICE FOCUSING ON SATISFACTION: 'DO WE PASS THE FRIENDS AND FAMILY TEST?'}

${ }^{1} \mathrm{MG}$ Semple, ${ }^{2} \mathrm{AC}$ Haigh. 'Faculty of Health and Life Science, University of Liverpool, Liverpool, UK; ${ }^{2}$ School of Medicine, University of Liverpool, Liverpool, UK

\subsection{6/archdischild-2020-rcpch.125}

Background The NHS Friends and Family Test (FFT) is routinely used to gather around 1.2 million pieces of feedback each month. This information is utilised to transform services in ways that matter most to patients. In 2015 there was a large drive to gain paediatric service user feedback. Despite this, a child friendly FFT is not available within large proportion of paediatric settings.

Aim This study aimed to provide a platform for children and young people to feedback on the services they use and to report this information back to the regional flexible bronchoscopy service providers, facilitating praise and suggestions for improvement.

Methodology Feedback regarding patient and parent/carer experience of the regional flexible bronchoscopy service was collected via an online questionnaire, with the main question based upon the NHS FFT. The online questionnaire was accessed via a website designed for this study. Adaptations to 